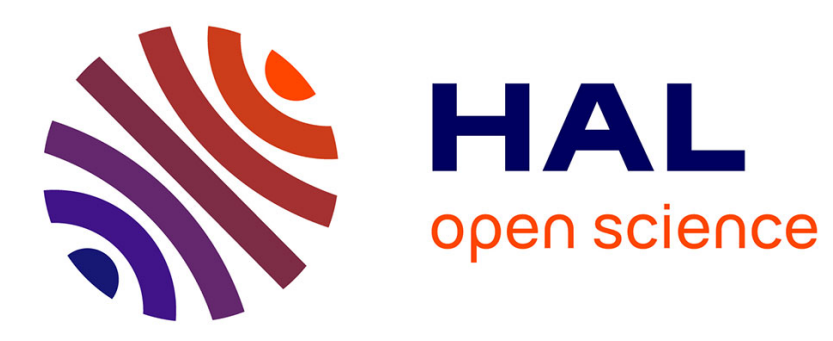

\title{
La prostitution des mineurs dans le débat républicain à la Belle Epoque. L'expertise juridique et l'échec d'une politique.
}

Pascale Quincy-Lefebvre

\section{- To cite this version:}

Pascale Quincy-Lefebvre. La prostitution des mineurs dans le débat républicain à la Belle Epoque. L'expertise juridique et l'échec d'une politique.. Histoire@Politique: revue du Centre d'histoire de Sciences Po, 2011, 2 (14), Non spécifié. 10.3917/hp.014.003 . hal-03439102

\section{HAL Id: hal-03439102 \\ https://univ-angers.hal.science/hal-03439102}

Submitted on 22 Nov 2021

HAL is a multi-disciplinary open access archive for the deposit and dissemination of scientific research documents, whether they are published or not. The documents may come from teaching and research institutions in France or abroad, or from public or private research centers.
L'archive ouverte pluridisciplinaire HAL, est destinée au dépôt et à la diffusion de documents scientifiques de niveau recherche, publiés ou non, émanant des établissements d'enseignement et de recherche français ou étrangers, des laboratoires publics ou privés. 


\section{La prostitution des mineurs dans le débat républicain à la Belle Epoque. L'expertise juridique et l'échec d'une politique.}

$\mathrm{Au}$ XIXe siècle, le libéralisme est le grand cadre de pensée des nouvelles logiques sociales ${ }^{1}$. La prostitution, parce qu'elle touche à l'intime, tout en empiétant sur le domaine public, est au cœur de profonds débats sur les registres de la contrainte dans un ordre libéral ${ }^{2}$. Quel mode de régulation pour une question en lien direct avec un possible gouvernement de la sexualité $?^{3}$ Au XIXe siècle, la prostitution est devenue une affaire de spécialistes. Jusque dans les années 1880, le sujet est dominé par les «scientifiques » ${ }^{4}$. Dans les décennies qui suivent, l'expertise évolue. Le changement se produit dans une société ralliée à la culture démocratique et chez des élites portant une attention plus marquée à la sexualité juvénile ${ }^{5}$. La production savante se spécialise. Fin XIXe siècle, à une époque où l'Etat est interpellé sur sa politique publique en matière de prostitution, la protection des mineurs devient un cheval de Troie pour un déplacement des expertises et des politiques ${ }^{6}$. L'indignation sur les jeunes est un possible appui dans la dénonciation de l'arbitraire administratif, pour l'émergence de la thématique légale et la conversion des principaux protagonistes (réglementaristes et abolitionnistes) à l'idée législative.

Le réglementarisme a défini un cadre d'intervention qui, à partir du second XIXe siècle, est au cœur de nombreuses polémiques. Le système a ses origines dans les pouvoirs confiés aux Municipalités en 1789. Il se précise à partir des années 1830 pour survivre jusqu'au milieu du XXe siècle. Pendant cette période, la réglementation de la prostitution signifie que cette activité est soumise à l'administration du préfet de police à Paris, à l'administration des maires en province. Encore au début du XXe siècle, aucune règle ne précise un âge minimal pour l'inscription sur les registres sanitaires. La Cour de cassation

\footnotetext{
${ }^{1}$ Voir sur les logiques de régulation à l'ère libérale, les études de l'historien canadien Jean-Marie Fecteau. Jean-Marie Fecteau, La liberté du pauvre. Sur la régulation du crime et de la pauvreté au XIXe siècle québécois, Montréal, VLB Editeur, 2004.

${ }^{2}$ Voir Les travaux de Deborah Gorham sur la prostitution des mineurs à l'ère victorienne.

${ }^{3}$ La discipline sexuelle s'est resserrée depuis le XVIIIe siècle à l'égard des jeunes avec un grand nombre d'écrits de pédagogues, de médecins comme l'a écrit Michel Foucault dans Histoire de la sexualité. Tome.I : La volonté de savoir, Paris, Gallimard, 1976.

${ }^{4}$ Amélie Maugère, préface de Janine Mossuz-Lavau, Les politiques de la prostitution du Moyen âge au XXIe siècle, Paris, Dalloz, nouvelle Bibliothèque de thèses de Science politique, 2009, p.84.

${ }^{5}$ Pour des travaux récents à propos des normes et politiques autour de la sexualité des jeunes depuis le XIXe siècle, voir V. Blanchard, R. Revenin, J.-J. Yvorel, dir., Les jeunes et la sexualité. Initiations, interdits, identités (XIXe-XXIe siècle), Paris, Editions Autrement, 2010.

${ }^{6}$ Sur les mutations du champ discursif autour de la prostitution, voir la thèse d'Amélie Maugère, op.cit.

Sur la chronologie du débat autour de la prostitution des mineures dans les années 1890 et 1900 (congrès, société générale des prisions, comité des enfants traduits en justice, Conseil municipal, assemblées parlementaires), voir la thèse de droit de Félix Lhose, La prostitution des mineures en France avant et après la loi du 11 avril 1908, Paris, Libr. Arthur Rousseau, 1913.
} 
admet qu'une mineure ne peut être mise en carte avant 16 ans $^{7}$. Parallèlement, la sexualité des mineures est de plus en plus cadrée par l'idée d'un âge en dessous duquel il ne peut y avoir consentement. A l'époque, les articles 330-335 du Code pénal de 1810 prévoient des causes aggravantes dans les cas d'attentat aux moeurs. L'article 331 mentionne les crimes de viol et d'attentat à la pudeur avec violence et l'article 332 précise que le coupable de ces crimes subira une peine aggravée, en l'occurrence les travaux forcés, si sa victime est « un enfant audessous de l'âge de quinze ans accomplis ». En 1832, une nouvelle loi introduit dans le droit pénal «l'attentat à la pudeur sans violence, ni contrainte» si l'enfant à moins de $11 \mathrm{ans}^{8}$. En 1863, le seuil est déplacé à 13 ans $^{9}$. La norme juridique se resserre mais l'historienne AnneClaude Ambroise-Rendu note à propos de la loi qu' « en discutant inlassablement de sa mise en œuvre la pratique judiciaire montrait également que cette définition était au fond bien loin d'être unanimement partagée. C'est bien pourquoi ce crime est demeuré constamment menacé, si l'on peut dire, de déqualification ${ }^{10}$. La loi apporte «sa pierre à l'édifice d'une définition de l'enfance, une enfance exclue a priori de la sexualité ${ }^{11}$. Ce n'est pas le cas pour l'adolescente dont la figure de dessine plus difficilement à l'époque. Certains s'en offusquent.

Alors que l'opposition entre réglementaristes et abolitionnistes a longtemps structuré le débat, début XXe siècle, les échanges se recomposent. Les réglementaristes cèdent le pas aux néo-réglementaristes; des rapprochements s'observent avec le mouvement des abolitionnistes, qui, comme l'ont montré Alain Corbin et Jean Michel Chaumont, accueille moins les défenseurs de la liberté individuelle que les « entrepreneurs de morale ». Du côté de l'expertise, au XIXe siècle, les médecins avaient été les hommes de savoir. Dans un contexte marqué par l'enjeu des libertés en démocratie, les juristes font une entrée remarquée. Des «mutations des répertoires discursifs savants » s'opèrent ${ }^{12}$. Appliquée aux mineurs, l'expertise légitime un nouveau regard. A plus long terme, le changement, né des discussions de l'entre-deux-siècles, initie de nouveaux modèles d'action publique. Derrière des formulations d'une extrême technicité, les juristes expriment l'ambition de traiter des «principes fondamentaux sur lesquels est ou devrait être fondé l'ordre social ${ }^{13}$.

\footnotetext{
${ }^{7}$ Anna Segretain, La loi du 11 avril 1908 sur la Prostitution des mineurs, maîtrise d'histoire sous la direction d'Eric Pierre, Université d'Angers, 2005.

${ }^{8}$ Anne-Claude Ambroise-Rendu « Attentats à la pudeur sur enfants : le crime sans violence est-il un crime ? (1810-années 1930) », Revue d'histoire moderne et contemporaine, n 56-4, 2009, p. 165-189.

${ }^{9}$ En 1945, il est relevé à 15 ans pour des relations hétérosexuelles mais à 21 ans dans les cas d'homosexualité.

${ }^{10}$ Anne-Claude Ambroise-Rendu, op.cit.

11 Ibid.

${ }^{12}$ Amélie Maugère, op.cit.

${ }^{13}$ Sur la littérature juridique, J.Commaille, L'esprit sociologique des lois, Paris, PUF, 1994, p.18.
} 
La scène retenue est celle des débats qui ont surgi lors de la «Commission pour l'étude du régime des mœurs ». La commission est réunie dans un contexte de crise : des scandales sur le fonctionnement de la police des mœurs et la dénonciation de cette zone de non droit qu'est alors le traitement administratif de la question de la prostitution en France. Un large consensus s'établit pour réformer les politiques publiques autour du fait prostitutionnel et supprimer la police des mœurs. Les experts sont convoqués. Pourtant, avant la Deuxième Guerre mondiale, la seule réforme importante est le vote de la loi du 11 avril 1908 sur les mineurs se livrant habituellement à la débauche ou à la prostitution ; une loi, au final, jugée inapplicable et quasi inappliquée.

La loi de 1908 n'a pas révolutionné la pratique judiciaire. Il n'empêche, les principes qui l'inspirent annoncent un changement de paradigme. Relire le roman de la loi, c'est s'intéresser à une approche qui, à la solution administrative ou pénale, substitue une intervention au civil et une norme de protection qui distingue la jeunesse. Les historiens se sont attardés sur les débats parlementaires ou sur l'échec de la loi concernant le traitement de la prostitution juvénile. Ici, nous proposons de revisiter la loi en amont du vote, par une étude qui s'attarde sur l'expertise qui a présidé aux orientations du texte. La place des juristes est centrale. Pourquoi ? Et avec quel impact sur le modèle de politique publique?

La décision de réunir une commission est prise dans l'immédiat contexte d'une campagne de presse ${ }^{14}$. Depuis plusieurs décennies, les abolitionnistes font entendre leurs voix dans des manifestations nationales et internationales. L'espoir est important de voir condamner le système réglementaire. A ses débuts, la commission rassemble jusqu'à 71 membres ${ }^{15}$. 36 séances sont tenues entre le 5 novembre 1903 et le 7 décembre 1906. Une sous-commission a en charge la réflexion sur les mineurs. Le scandale a donné la parole aux experts, et, parce que la question soulevée par la presse a concerné la question des libertés publiques et de l'égalité devant la loi, des juristes ont été convoqués. Un homme se distingue tout particulièrement. Si son frère a une renommée certaine, ce n'est pas le cas de Gustave Le

\footnotetext{
${ }^{14}$ Pour une description des scandales par un abolitionnistes, voir Louis Fiaux, Un nouveau régime des mæurs. Abolition de la police des mours. Le régime de la loi, Paris, Félix Alcan, 1908, 512p. Par exemple le scandale autour de l'arrestation d'un publiciste M.A Forissier de La Lanterne. Au cours d'une promenade avec sa fiancée et une amie de celle-ci, il aurait été pris à partie par deux agents de la police des moeurs. Ivre pour l'un. Dans un premier temps, le policier est couvert par ses collègues (faux témoignages pour accuser le publiciste de racolage...). Après une contre enquête, les policiers sont condamnés.

L'affaire intervient après d'autres «scandales » comme le suicide de prostituées cherchant à échapper à un contrôle sanitaire particulièrement traumatisant.

Sur la campagne contre la police des mœurs, voir J. M. Berlière, La Police des mours sous la IIIe République, Paris, Seuil, 1992.

${ }^{15}$ Louis Fiaux, op.cit. Mais ils sont 73 pour J.M. Berlière, op.cit. p.155.
} 
Poittevin. Le succès des approches qu'il défend devant la commission indique le sens collectif du changement autant qu'il informe sur la ténacité d'un homme. Le débat qui l'oppose à un autre juriste et homme politique éminent, René Bérenger, dit les divisions sur un nouveau champ légal d'intervention auprès des mineurs : le sexe.

\section{La République, le sexe et les jeunes. La parole aux experts.}

La prostitution n'est pas un délit dans le droit français. Elle est néanmoins saisie comme un désordre justifiant le développement d'un contrôle policier et administratif bien étudié par l'historien Alain Corbin ${ }^{16}$. Comme pratique réglementée, la prostitution enferme la jeune fille ou la jeune femme dans un état. La mise en carte des « filles soumises » symbolise une gestion sanitaire et policière d'une population à risque. Lorsqu'elle concerne des jeunes filles mineures, la pratique administrative a pu être dénoncée comme un encouragement à la prostitution mais également comme un acte surplombant la puissance paternelle.

A la charnière des deux siècles, la question est débattue lors des congrès et dans des revues spécialisées. Des figures du «philanthropisme juridique » se mobilisent dans le cercle des patronages et des comités de défense des enfants traduits en justice. A Paris, les forces de police sont invitées à orienter les mineures vers des œuvres ou à les déférer devant les tribunaux afin de procéder, par voie pénale, à leur «préservation ». Dans ce second cas, la mineure, inculpée pour vagabondage, peut faire l'objet d'une mesure d'éducation correctionnelle pour avoir agi «sans discernement» (application de l'article $66 \mathrm{du}$ Code pénal). Mais, comme la présence des jeunes prostituées n'est guère appréciée dans les établissements de réforme ou «maisons pénitentiaires », elles sont, le plus souvent, au grand dam des belles âmes, condamnées à de courtes peines de prison, pour se retrouver très vite dans la rue.

Jusqu'en 1908, la situation des mineures prostituées ne fait l'objet d'aucune loi spécifique. Une sensibilité nouvelle a pourtant surgi pour faire reconnaître la protection de l'enfance en danger comme un objet des politiques publiques. Dans les années 1880, le médecin, philanthrope et sénateur Théophile Roussel, est un des premiers réformateurs qui, par la loi, cherche à individualiser et à protéger une population que beaucoup pensent corrompue et incorrigible. A cette époque, l'homme, qui a inspiré la loi de 1874 sur la protection des enfants chez leurs nourrices, propose d'assimiler la prostitution des mineures à

\footnotetext{
${ }^{16}$ Alain Corbin, Les filles de noce. Misère sexuelle et prostitution aux XIX et XXe siècles, Paris, Aubier, 1978.
} 
un abandon moral ${ }^{17}$. La logique est double : prévention du crime et protection des plus faibles. Lorsque la grande loi de 1889 sur la protection des enfants maltraités ou moralement abandonnés est enfin votée, la proposition n'est pas reprise. Une brèche se dessine pourtant en 1898. La loi sur la répression des violences, voies de fait, actes de cruauté et attentats commis envers les enfants réunit, dans un même texte, des enfants auteurs et victimes de délits. Elle ouvre le champ judiciaire de la protection au mineur victime de ses propres actes. La loi est largement l'œuvre d'un sénateur, René Bérenger. L'homme est une figure de choc des campagnes morales contre la pornographie. Il est également un actif militant de la lutte contre « la traite des femmes blanches ».Cette dernière affaire devient une cause. Comme à propos des enfants, elle introduit dans le champ de la prostitution, des images fortes de victimes à partir desquelles produire un discours moral.

En cette fin de siècle, au sein même du milieu de la « réforme », la nécessité d'une loi ne fait pas l'unanimité. Le sujet est bien souvent présenté comme délicat. Pour certains, le progrès doit venir d'une loi susceptible d'utiliser le critère de l'âge pour déclarer la prostitution des mineurs illégale et justifier une action publique de protection. Pour d'autres, il suffit d'avoir des garanties pour que les juges puissent continuer à utiliser un artifice juridique et inculper les mineures pour vagabondage. Le débat sur la police des mœurs crée une actualité nouvelle. Il est le moment choisi pour reprendre le dossier à deux niveaux. Le premier concerne la prostitution en général. Les scandales sont utilisés pour faire le procès du réglementarisme. Le second a trait à la situation des prostituées mineures. La dernière question présente l'avantage de réunir, sur bien des propositions, abolitionnistes et néoréglementaristes. La situation des mineures autorise un déplacement des expertises qui, à terme, introduit un autre paradigme dans le traitement de la prostitution et des prostituées dans les politiques publiques.

L'exploitation médiatique de différentes affaires mettant en cause la police des mœurs donne une tribune aux abolitionnistes. L'historien Jean Marc Berlière a décrit la place des scandales dans la démocratisation du débat. Face à l'indignation, les politiques s'imposent de réagir. Ils le font par une première décision : la réunion d'une commission. Il importe de créer un lieu pour l'expertise afin d'asseoir la fabrique de la loi sur les savoirs experts. La réactivité des politiques n'est pas étrangère à la culture radicale. Le contexte est celui de l'après affaire Dreyfus. L'époque est favorable à l'expression d'un discours sur la défense des libertés

\footnotetext{
${ }^{17}$ Jean-Jacques Yvorel, «Légiférer sur la sexualité de la jeunesse. La loi de 1908 sur la prostitution des mineurs », in Annie Stora Lamarre, dir., La cité charnelle du droit, Besançon, Presses universitaires franccomtoises, 2002, p.109-127.
} 
publiques et condamnant l'arbitraire. L'avènement, puis l'affirmation de la République radicale ouvrent des espaces nouveaux à des façons de penser et de faire du «social » dans le cadre des politiques publiques. En quête d'une troisième voie entre libéralisme et socialisme, des hommes de pouvoir sont susceptibles d'accueillir d'autres expertises pouvant justifier une autre place pour l'Etat dans des logiques davantage tournées vers l'intégration des masses, ces anciennes «classes dangereuses». Clemenceau est au pouvoir; la grande figure du radicalisme, par ailleurs, homme de presse, est un homme engagé sur le sujet de la prostitution. Les abolitionnistes ont bon espoir de faire triompher leur point de vue. Au final, les seules dispositions qui débouchent, avant guerre, sur un changement de la loi sont celles concernant les mineurs.

Lorsqu'il s'agit de désigner les membres de la nouvelle commission, la décision est prise de réunir des représentants de chaque camp idéologique ${ }^{18}$. La composition fait une place à des politiques, à des hauts fonctionnaires, à des publicistes. Le gouvernement fait appel à des experts, désignés comme tel, pour un dossier présenté comme délicat, dans une affaire que les politiques ont longtemps refusé de débattre par «pudeur» ou intérêt. Les abolitionnistes sont optimistes; ils pensent la suppression de la police des mœurs quasi acquise. Les témoignages se succèdent et le propre chef de la police parisienne, le préfet Lépine, rallie certaines de leurs positions. Le débat se veut global. Une des questions soulevées concerne une politique pour les mineures. Dans un premier temps, il s'agit bien de traiter de l'unique situation des filles, l'intégration des garçons intervenant tardivement dans la discussion.

La caution de l'expert est recherchée alors que, derrière la prostitution, il importe de légitimer un contrôle sur les comportements sexuels de certains jeunes. La question va bien au-delà de la police des mœurs. Le projet élaboré dans le cadre de la commission s'inscrit dans un mouvement qui a comme balises les vœux votés lors de différents congrès ou dans le cadre des comités ou sociétés impliqués dans la réforme de la protection des mineurs. La question, parce qu'elle traite de sujets mineurs, déborde le périmètre des définitions classiques de la prostitution. L'expertise a vocation à légitimer des glissements dans des projets qui traquent les expressions d'une sexualité qualifiée d'excessive des jeunes filles ou de dénaturée chez les garçons (homosexualité). La problématique n'est pas seulement sanitaire, elle est morale. De même, l'enjeu n'est pas l'unique protection de la jeunesse mais l'état de la nation.

\footnotetext{
${ }^{18}$ Pour les réglementaristes, la prostitution est perçue comme un mal nécessaire. La réglementation (le fichier sanitaire...) permet de contrôler et de contenir le phénomène.

Les abolitionniste s'opposent au système réglementaire au nom de la liberté de disposer de soi même. Certains sont tentés de supprimer la prostitution qui avilit la femme.
} 
L'élargissement du territoire qui, de la prostitution, s'étend à «la débauche » heurte des principes du droit. Les questions sont nombreuses. Le débat a été fort long. Le problème majeur est de concilier le respect des libertés individuelles et les impératifs de protection. Une réponse est attendue des spécialistes des sciences juridiques.

\section{Pour une nouvelle méthodologie ${ }^{19}$.}

Les débats du second XIXe siècle sur la prostitution ont privilégié l'angle sanitaire. Le système réglementariste s'est solidifié à travers un discours médical. Le risque contre lequel la société devait d'abord se protéger était celui de la syphilis dans un état des savoirs où cette infection était une cause de stérilité et était vue comme un facteur de dégénérescence de la race. Face à la prostitution, mal social mais institution nécessaire pour nombre de libéraux, la question était donc d'abord celle du contrôle sanitaire des prostituées.

Dans ce premier contexte, hommes de sciences et de progrès, les médecins ont exploité leur position d'experts pour asseoir leur profession et développer des ambitions politiques. Puis l'expertise a pu déboucher sur un militantisme. Parce que touchant la question des libertés, le statut de la prostitution renvoie à des positionnements philosophiques et politiques qui divisent le corps médical. Au moment de la commission, Louis Fiaux est l'image même de ces médecins engagés. Conseiller municipal à Paris, chef de file des abolitionnistes, il recherche la collaboration des juristes et il valorise leur position d'experts dans un débat où la question sanitaire est désormais seconde.

L'ouverture la plus spectaculaire se fait sur le terrain de la protection des mineurs. Louis Fiaux prend publiquement la parole pour présenter la pluridisciplinarité de la commission extraparlementaire comme la méthode à suivre pour élaborer les nouvelles politiques. La prostitution est présentée comme un problème global où «tout se tient, moralité, légalité, ordre extérieur, médecine publique ». Le médecin en appelle à une nécessaire «fusion des esprits $»^{20}$. Il estime que la commission a trouvé celle-ci dans la collaboration avec des juristes. Alors que le juge Gustave Le Poittevin s'apprêtait à être un expert parmi d'autres, le contexte politique le pousse sur le devant de la scène.

\footnotetext{
${ }^{19}$ La commission a été instituée par décret du 18 juillet 1903. La $1^{\text {ere }}$ séance a lieu le 5 novembre 1903.

${ }^{20}$ Louis Fiaux, op.cit., p.395-396. Le militant abolitionniste date de la conférence de Bruxelles (1899) portant sur la prophylaxie de la syphilis, l'ouverture de l'expertise à d'autres acteurs que des médecins. La conférence est présentée comme ayant montré la voie pour une nouvelle méthodologie. Pour le même auteur, c'est la commission extraparlementaire qui « brisa totalement » le monopole des médecins.
} 
Lorsque la décision est prise de réunir une commission, le juriste ne saisit pas de suite les nouveaux enjeux autour de son domaine de compétence. Les scandales autour de la police des mœurs ont précipité la réponse publique. C'est dans l'urgence que la composition de la commission est élaborée. Gustave Le Poittevin n'est pas une figure éminente de la science juridique de l'époque. Il est bien moins connu que son frère Albert Léon, grand professeur de droit à Paris et contributeur régulier à la Société générale des prisons ou autre instance de régulation du droit et de la morale juridique. Hasard ou surprise ? L'homme est absent à la séance d'ouverture de la commission. Il ne tarde pas néanmoins à en devenir un personnage clé. En particulier, il est nommé rapporteur de la sous commission en charge de la préparation d'un projet de loi sur la prostitution des mineurs. L'homme a la bonne surprise de découvrir dans la commission, un espace qui sollicite son expertise. Largement étranger au milieu qui, traditionnellement, débat de ces questions, il saisit la perche qui lui est tendue. Son rôle dans la commission dit l'intérêt déjà marqué d'une profession pour l'expertise. Il est également l'expression d'un engagement plus personnel ${ }^{21}$.

Gustave Le Poittevin est un provincial monté à Paris. Il a pu manifester de grandes ambitions à l'exemple de son frère Alfred Léon Le Poittevin. Mais lorsque l'affaire de la commission débute, le juge d'instruction apparaît davantage comme un homme bloqué dans sa carrière ${ }^{22}$. Au début du siècle, il est connu pour ses traités de procédure ${ }^{23}$. L'homme a une réputation de laborieux. Au palais, un surnom lui a été donné, celui de «Bénédictin du parquet » afin de souligner l'ampleur et la minutie de son travail. Lorsqu'il est sollicité pour faire partie de la commission, il n'est pas connu comme s'intéressant à la question de la prostitution ou des mineurs. Ce n'est pas le cas de son frère qui, comme figure de la société générale des prisons, a participé à différents débats sur la justice et la protection des mineurs. Sans avoir de compétences particulières dans le champ visé par la commission, il est néanmoins connu par l'intérêt qu'il porte à l'expertise. Dans des confidences à sa petite fille, le juriste reviendra sur la place qu'elle a prise dans sa carrière. Au soir de sa vie, il lui confiera

\footnotetext{
${ }^{21}$ Madeleine Bonnelle Gustave Le Poittevin (1856-1930), dactyl, 1976, 61p. L'auteure est la petite fille du juriste. Le document est conservé à la B.N.

22 Le magistrat est appelé à Paris en 1894 comme substitut du Procureur de la République. En 1896, il est nommé Juge au Tribunal de la Seine. Fonction qu'il occupe jusqu'en 1908.

${ }^{23}$ Pour une présentation des travaux de G.Le Poitevin, Madeleine Bonnelle, op.cit.

Parmi ses études :

1899 , Traité des Casiers Judiciaires

1902, Traité de la Presse » couronné par l'Académie des Science morales et Politiques

1904, Dictionnaire-Formulaire de Simple Police

1905, Traité de Droit Pénal Militaire, en collaboration avec le Colonel Augier

A cette époque il se voit offrir le lourd travail d'annoter le « Code d'Instruction Criminelle » pour la collection Sirey tandis que son ami Emile Garçon annote le Code Pénal. Le premier volume sort en $1911 \ldots$
} 
toute sa satisfaction d'avoir été le conseiller de plusieurs ministères et, par son rôle de rapporteur dans différentes commissions, d'avoir «parfois pu faire évoluer la loi. ${ }^{24}$. Chez l'homme, la satisfaction a également un goût de revanche ${ }^{25}$. Il s'est servi de l'expertise comme moyen de faire valoir son mérite personnel alors qu'il jugeait que sa carrière de magistrat était trop soumise à la volonté du prince. Fantasme ? Gustave Le Poittevin, parce que catholique, mais aussi parce qu'il est celui qui a instruit le dossier de Panama, est convaincu qu'il est la bête noire de personnalités radicales au pouvoir. Il lui faut donner des gages à la République pour avancer dans la carrière. Juge d'instruction au tribunal de la Seine (et bientôt doyen dans cette charge), il aspire à devenir Conseiller à la Cour d'appel ou, mieux encore, à la Cour de Cassation. L'expertise est la voie qu'il entreprend dès lors de privilégier. Avec le temps, la stratégie sera payante. Il accédera à la Cour d'appel avec les fonctions de Vice-président puis de Président. Un petit bémol est à noter dans le plan de carrière qu'il envisage dans les années 1900 : il ne sera jamais appelé à siéger à la Cour de Cassation.

Comme expert, le juriste va mettre toute sa science de la procédure au service de la commission. Lors des travaux de la commission, son action est saluée pour sa rigueur. Durant ces mêmes travaux et une fois la loi votée sur la prostitution des mineurs, elle a pu être également présentée comme à l'origine de la complexité et de l'échec de la loi.

\section{L'expert et le politique}

La commission va débattre amplement de la question des mineurs. L'expertise est «scientifique ». Sur le plan des risques sanitaires et de santé publique, la prostituée mineure est présentée comme la plus dangereuse. Susceptible de ne pas être encartée, donc contrôlée, elle est décrite comme un vecteur important de transmission des maladies vénériennes. Pourtant, les personnalités conviées prennent le parti de ne pas rester sur ce champ principal de lecture de la déviance. L'expertise va se faire «morale». La prostituée mineure est assimilée à une personne en danger. Les politiques sont invités à mettre légalement en place des mesures de protection dites de «redressement moral» propres à la relever tout en l'empêchant de nuire.

\footnotetext{
${ }^{24}$ Autre expérience qui a particulier marqué le juriste, le rôle joué en 1923 comme rapporteur de la Commission pour la refonte du Code de Justice pour l'armée. Il note alors : «c'est fort important d'être un tel rapporteur, parce que son nom reste attaché à l'oeuvre. Ibid. p.28-29.

${ }^{25}$ Plus encore après 1908 lorsqu'il eut à subir une destitution à la suite de la fuite d'un escroc lors d'une mesure de libération provisoire qui devait contribuer à l'enquête. La décision fut communiquée à la presse. La destitution intervient alors qu'il devait passer Conseiller à la Cour au bénéfice de l'ancienneté. Le magistrat interpréta la sévérité de Briand comme une nouvelle vengeance des « Panamistes ». Madeleine Bonnelle, op.cit.
} 
L'évolution n'est pas étrangère à un mouvement de fond sur les représentations de l'enfance dans une démocratie. La définition d'une citoyenneté active a justifié un resserrement du discours sur la figure du mineur comme incapable et potentiellement non discernant au pénal. Les idées libérales et la croyance dans le progrès font de l'enfance et, partiellement, de la jeunesse, un désir d'avenir. Les hommes de la Troisième République investissent sur l'instruction des enfants du peuple comme gage de paix et de progrès social. Symboliquement, ils ont également fait sauter le verrou juridique de la toute puissance paternelle si la conduite des parents met en danger l'enfant. Sur le plan des normes, le droit pénal demeure le principal outil de régulation des conduites déviantes. Dans ce cadre, les juges sont invités à utiliser avec parcimonie les peines de prison au profit de mesures d'éducation correctionnelle dans des établissements à la discipline toute carcérale. Par ailleurs, comme l'a montré Alain Corbin, on assiste à la sortie croissante des filles et des femmes du judiciaire. L'évolution est controversée lorsqu'il s'agit de mineures. Le déplacement de l'âge de la majorité pénale de 16 ans à 18 ans adopté au même moment, dit la volonté de contrôler et de rééduquer une jeunesse dans un cadre à singulariser ${ }^{26}$. La protection est également l'argument des experts qui, en 1905, sont réunis pour traiter de la prostitution. Dans la Commission, si le consensus s'exprime sur la légalisation des mesures, le débat est vif sur le périmètre de la loi et la procédure. Une première proposition est avancée par le sénateur Bérenger. Elle est rejetée à la suite de la contre proposition de Le Poittevin ${ }^{27}$. C'est cette dernière qui servira de base au projet de loi présenté par le gouvernement et adopté en 1908.

Paradoxe, le fameux sénateur Bérenger, un homme né en 1830, est également un juriste. Néanmoins, face à l'expert Le Poittevin, l'homme se positionne d'abord en politique. Le « père la pudeur », bien connu pour ses interventions à la chambre et dans la presse contre la pornographie, a été, précédemment, l'auteur d'une proposition de loi en 1894 sur la prostitution et les outrages aux bonnes mœurs. L'article 4 concernait les filles mineures. Comme d'autres propositions, le texte n'a pas débouché sur une loi nouvelle. L'homme a plus de succès avec le vote de la loi de 1898 sur la répression des violences, voies de fait, actes de cruauté et attentats commis envers les enfants. Ce dernier texte a un impact majeur puisque,

\footnotetext{
${ }^{26}$ Sur la loi du 12 avril 1906 fixant à 18 ans l'âge de la majorité pénale avec des dispositions particulières pour les 16-18 ans, voir, Pascale Quincy-Lefebvre, « Droit, régulation et jeunesse. Réforme de la majorité pénale et naissance des 16-18 ans à la Belle Epoque », in Ludivine Bantigny, Ivan Jablonka, dir., Jeunesse oblige. Histoire des jeunes en France XIXe-XXIe siècle, Paris, PUF, 2009, p. 95-110.

27 Les comptes-rendus des séances ont été publiés. Commission extraparlementaire du Régime des mœurs, Procès verbaux des séances, Melun, Imprimerie administrative, 1909. Le Poittevin propose des amendements, véritable contre proposition au projet Bérenger lors de la 24ème séance, le 23 juin 1904. P.588 et suivantes. Le principal argument est alors que «la proposition telle que la présente M.Bérenger est en contradiction avec les principes de notre droit public qui garantissent le respect de la liberté individuelle et de la puissance paternelle ».
} 
par un subterfuge qui a échappé aux parlementaires, il a permis qu'un mineur, auteur d'un délit mais considéré comme en danger, puisse faire l'objet d'une mesure de protection afin d'être confié à une œuvre ou à l'Assistance publique. Homme d'expérience, le sénateur inamovible connait la fabrique de la loi et il a une bonne connaissance des mœurs des parlementaires. Il est aussi un homme de convictions et de réseaux.

Les historiennes, Martine Kaluszynski dans ses travaux sur la Société générale des prisons, et Annie Stora-Lamarre dans ses ouvrages sur les censeurs de la IIIème République, présentent Bérenger comme animé d'une grande ambition: celle de rénover la moralité publique et d'imposer le respect des bonnes mœurs. Ce républicain catholique attaché au «devoir social » défend un ordre moral ${ }^{28}$. Lorsqu'il est appelé à siéger à la commission extraparlementaire, il a une stature bien supérieure à celle de Le Poittevin. René Bérenger a été le fondateur de la Société générale des prisons, laquelle a fonctionné durant plusieurs décennies sur un modèle naviguant entre la société savante et le rôle d'une commission extraparlementaire. Dans les années 1880 et 1890, la société a même pu être comparée a un «gouvernement privé des juristes ${ }^{29}$. Au début du siècle, le sénateur est membre de l'Académie des sciences morales et juridiques. Depuis 1904, il est le Président de la Société d'économie sociale. Par ailleurs, il préside aux destinées de la Ligue contre la licence dans la rue. En 1905, il est également le Président des Sociétés de patronage de France et il est, bien entendu, un des fondateurs de l'Association pour la répression de la traite des blanches et la préservation de la jeune fille ${ }^{30}$. Sa proposition de loi a été votée par le Sénat en 1894 mais a été bloquée par les élus de l'autre chambre. L'éclatement des scandales autour de la mondaine et la mise sur pied de la commission extraparlementaire sont des opportunités à ne pas manquer pour une solution légale. L'inertie est rompue. Mais le jeu s'avère plus compliqué, la sous-commission devenant un lieu d'affrontement entre l'autorité du politique et celle de l'expert.

\section{Deux projets dans la balance}

\footnotetext{
${ }^{28}$ Sur René Bérenger, voir B.Schnapper, "Le sénateur Bérenger et les progrès de la répression pénale en France, 1870-1914", Annales de la Faculté de droit d'Istanbul, n 48, 1979, repris dans Voies nouvelles en histoire du droit, la justice, la famille, la répression pénale (XVIe-XXe siècles), Paris, PUF, 1991, p. 355-373. Mais également les développements d'Annie Stora-Lamarre, de Jacques Bourquin. Sur Bérenger et sur les juristes comme groupe socio-professionnel, le rapport de recherche de Martine Kaluszynski, Les Hommes de la Société générale des prisons (1877-1900), CERAF, 1993.

${ }^{29}$ Martine Kaluszynski, «Savoirs et politique sur le crime au XIXe siècle. La morale comme réponse à la question pénale », in B.Garnot, dir., Ordre moral et délinquance de l'antiquité au XIXe siècle, Actes du colloque de Dijon 7 et 8 octobre 1993, Editions Universitaires de Dijon, 1994, p.83-94.

${ }^{30}$ Annie Stora-Lamarre,L a République des faibles, Paris, A.Colin, 2005, p.89.
} 
Contrairement à Le Poittevin, le sénateur Bérenger a déjà travaillé sur la question des prostituées mineures. Il intervient dans la commission, armé d'un projet qui reprend des points précédemment discutés dans le monde du «philanthropisme juridique ». L'homme défend l'impératif de la loi. Il est urgent de sortir cette population de l'arbitraire (le contrôle et les internements administratifs). Il importe de se saisir d'une indignation, celle de ceux qui semblent découvrir que des filles mineures peuvent être encartées ou se retrouver incarcérées aux côtés de prostituées majeures qui ne pourront que les pervertir un peu plus.

Bérenger impose comme objectifs à la loi d'arrêter le spectacle de la prostitution des mineures sur la voie publique et de fixer les cadres d'une réforme des jeunes filles. Figure du néo-réglementarisme et catholique, il se dit opposé aux thèses lombrosiennes de l'école anthropologique italienne sur les criminels-nés ${ }^{31}$. L'homme de la grande loi de 1891 sur le sursis est plus proche d'un autre catholique, Raymond Saleilles, professeur de droit et auteur en 1898, d'un ouvrage appelé à être réédité, L'individualisation de la peine. Etude de criminalité sociale. Bérenger justifie une loi sur les prostituées mineures par leur dangerosité pour l'ordre public mais également par le relèvement possible de celles qui sont « tombées ». Il est l'homme qui, le premier dans la commission, pose le principe de la protection morale et rééducatrice à partir de laquelle légiférer sur la prostituée mineure.

Dans l'échange qui l'oppose à Le Poittevin, Bérenger défend un projet qui prend pour seule cible «les mineures de 18 ans saisies en état habituel de prostitution sur la voie publique » et, à ce titre, objet de désordre au regard des «bonnes mœurs ». Le parlementaire juge contraires aux attentes de la population des initiatives dans le champ de la sphère privée alors que le père a autorité sur sa fille. Se voulant pragmatique, il présente sa proposition comme la plus à même d'obtenir l'adhésion des parlementaires. Il importe que la loi passe et que la prostituée mineure fasse l'objet d'une politique publique de mise à l'écart et de relèvement. Le sénateur se montre très attaché à la simplicité de la procédure menant la déviante vers son juge. Le rôle majeur est confié au juge de paix, magistrat symbole de proximité. La procédure doit être simple pour être socialement, moralement efficace alors que l'urgence est là. Le projet prévoit une saisie par les agents de la force publique. Après décision du juge de paix, la jeune fille pourra être envoyée dans un établissement de réforme.

Dans la foulée de la présentation, Gustave Le Poittevin prend l'initiative d'une contreproposition. Une formule de l'expert résume la nature principale de sa critique. En prenant

\footnotetext{
${ }^{31}$ La question des mineures est abordée, pour la première fois, par les membres de la commission lors de la 11 ème séance, le 20 décembre 1904.
} 
soin de rappeler que, dans le code français, la prostitution n'est pas un délit, le projet de Bérenger reviendrait à créer «contre la mineure un délit d'ordre civil » ${ }^{32}$. Il a plusieurs points de divergence avec la projet de Bérenger. Le premier est de ne pas limiter l'action du juge à la prostitution des mineures sur la voie publique. Alors que le sénateur en appelait aux bonnes mœurs, l'expert se place résolument dans le cadre de la protection du mineur en danger. Qu'importe le lieu puisque c'est de la mineure que la loi doit se préoccuper. La loi de 1889 sur la déchéance paternelle a ouvert une brèche, elle est le modèle à suivre pour penser l'action publique en direction des prostituées mineures. La proposition de Bérenger est jugée mal adaptée à ce que doit être une politique publique dans le domaine de la protection des mineurs en danger. Dans son premier texte, la prostituée est la mineure qui «se donne sans choix et au premier venu, avec ou sans idée de gain $»^{33}$. Le droit doit pouvoir intervenir là où il y a «abus prématuré, dégénéré en habitude des rapports sexuels avec le tout venant ${ }^{34}$. En juriste érudit, Le Poittevin justifie la formule « avec ou sans idée de gain » en renvoyant à la définition de la prostitution des jurisconsultes romains. Elle lui semble juridiquement plus défendable qu'une formule autorisant le tribunal à intervenir dans les cas de «débauche ${ }^{35}$. L'argutie ne change pas le projet: présenter devant le juge et faire interner des mineures faisant preuve d'une sexualité déviante, quelque soit le lieu. Ainsi, le juriste met sa science au service d'une politique plus intrusive dans le champ des comportements privés ${ }^{36}$. Les grandes lignes se retrouvent dans le projet de loi du gouvernement qui distingue néanmoins la débauche de la prostitution. Lors du vote, des sénateurs proposent d'étendre les mesures à une population encore plus large, celle des mineurs donnant de «très graves sujets de mécontentements ». L'amendement n'est pas retenu ${ }^{37}$.

Avec Bérenger, les divergences portent sur les registres de légitimité d'une action légale mais également sur la procédure. Le Poittevin se pose en défenseur du droit positif. Le juriste reproche à Bérenger, une procédure trop expéditive en contradiction avec les principes

\footnotetext{
${ }^{32}$ Commission extraparlementaire du Régime des mœurs, Procès verbaux des séances,op.cit.,p.590.

${ }^{33}$ Ibid.

${ }^{34}$ Ibid., 30 nov 1906, p. 922.

35 L'article 2 de la loi du 11 avril 1908 sur la prostitution des mineurs permettra au père ou à la mère d'un mineur de 18 ans qui se livre à la débauche de faire appel au tribunal civil pour qu'il soit placé dans un établissement ad hoc. La débauche est dans ce sens une tendance à la concupiscence dépourvue de finalité vénale. Jean Jacques Yvorel, op.cit., p.109-127.

${ }^{36}$ Sur cette intrusion croissante, au nom de la protection des mineurs, dans la sexualité des jeunes, voir la préface et les articles réunis par Christine Machiels et Eric Pierre, dans le numéro «La prostitution des mineur(e)s au XXème siècle », RHEI, ${ }^{\circ} 10$, octobre 2008.

${ }^{37}$ Chambre des députés, séance du 23 mars 1908. Parmi les opposants figure le député Paul Meunier qui souligne le danger à mélanger «indociles » et «pervertis ». Plutôt que de prolonger les discussions si un amendement est déposé, le rapporteur supprime la formule et présente le texte du gouvernement.
} 
qui garantissent le respect des droits individuels. Sous sa plume, Bérenger est l'homme de la décision «bienveillante » mais « rapide » là où il faut une décision «bienveillante et sans erreur $»^{38}$. La justice ne doit pas se tromper. Par exemple, la jeune qui a flirté ne doit pas être confondue avec la débauchée. Comme la prostitution n'est pas un délit, la procédure doit respecter la liberté de la jeune fille et l'autorité du père de famille. La contre-proposition confie au tribunal civil et non au juge de paix, la mise en œuvre de la loi (avec appel possible de la décision). Parce que l'action est au civil et non pas au pénal, la jeune fille ne doit pas être traitée comme une «coupable ». Elle ne peut être «saisie » par les forces de police mais elle doit être appelée à comparaître. La science juridique est obligée de reconnaître dans le ou la mineur[e] un sujet de droit. La pratique ne doit pas transiger avec les grands principes du droit et les libertés fondamentales doivent être respectées. Enfin, Le Poittevin revient sur les conditions d'internement. Comme la prostitution n'est pas un délit, les établissements pénitentiaires sont inadaptés. Des maisons de réforme ou «établissements spéciaux » devront être ouverts et pouvoir être contrôlés par les pouvoirs publics puisqu'ils retiennent les mineurs sur décision judiciaire. L'Etat devra veiller, en particulier, à ce qu'un pécule, proportionnel au travail fourni, leur soit versé.

L'expertise de Le Poittevin a cela d'étrange qu'elle cherche à concilier les canons du droit positif avec les aspirations des «entrepreneurs de morale» mais aussi celles des représentants de la République radicale. Son expertise est saluée par les abolitionnistes et, à l'exception de Bérenger, par les néo-réglementaristes. A l'image de ce que produit l'époque, le droit sert à importer un répertoire moral sur une question, la prostitution, jusque là dans les mains des experts scientifiques. Le registre «moral » annexe les avancées discursives sur des questions comme celles de la traite des femmes et les nouveaux standards de la protection des mineurs en danger ${ }^{39}$. Sous couvert de condamner chez Bérenger, un texte trop conservateur dans son approche de la prostitution (les bonnes mœurs et l'ordre public), Le Poittevin entend démontrer que le droit positif peut accueillir la greffe.

Le sénateur Bérenger tente de mettre en garde la commission sur le côté irréaliste de la contre-proposition de son collègue. Dans son argumentaire, il se montre prudent dans la défense d'un droit de «la défense sociale » et des idées de Saleilles. Le sénateur préfère apposer son expérience de politique. L'orateur supplie la commission « de mettre le projet actuel un peu en harmonie avec le tempérament d'une assemblée parlementaire »; il l'invite à

\footnotetext{
${ }^{38}$ Louis Fiaux, op.cit., p.344.

${ }^{39}$ Amélie Maugère, op.cit., p.84.
} 
«ne pas effaroucher les parlementaires par un texte qui ne s'appuierait pas sur la seule question de l'ordre public, un texte compliqué et coûteux dans son application ». Toujours sur un ton policé qui sied à l'échange entre des hommes de savoir, le sénateur dit espérer que «son excellent collègue et ami M. Le Poittevin lui permettra de dire qu'il a peut être été entraîné trop loin par sa profonde science juridique, qu'il s'est montré, peut être un peu trop professeur de droit $»^{40}$.

Bérenger ne convainc pas. Le Poittevin a pour lui une assiduité remarquable dans un processus qui s'étire sur des mois. Le professionnalisme rassure les quelques autres figures qui, à l'inverse de beaucoup d'autres, n'ont pas délaissé les sièges de la commission. Par ailleurs, l'homme a su utiliser avec habilité l'argument qui fait mouche dans le contexte qui suit l'affaire Dreyfus : la protection des droits de la personne. En faisant de la protection de cette même personne, l'angle des politiques sur le terrain de la prostitution, il produit un texte susceptible de satisfaire les abolitionnistes et les néo-réglementaristes. Lors du vote, sur onze présents, dix apportent leur suffrage à sa proposition.

Les membres de la commission se prononcent sur un texte qui, au final, porte sur les deux sexes. A l'origine, la commission a été rassemblée pour discuter d'un projet concernant la seule prostitution féminine. L'élargissement n'a pas fait l'objet de discussion parmi les experts de la commission. L'évolution a été imposée de l'extérieur, en 1906. Des parlementaires n'ont pas caché leur malaise d'avoir un texte qui supposait que la prostitution pouvait également se décliner au masculin. Quant aux experts, ils ont plutôt eu le souci de hiérarchiser les priorités. Le commentaire du médecin Louis Fiaux est éclairant : «Evidement de ces deux groupes, l'un, celui des filles, est incomparablement beaucoup plus considérable que l'autre, et il n'y a, ni numériquement, ni même moralement, aucun rapprochement à faire entre eux : mais Monsieur le Procureur général Bulot...a pesé de toute son influence sur la commission pour l'amener à étendre ses mesures de protection aux mineurs de «l'autre sexe »». Lors de la séance du 30 novembre 1906, Le Poittevin, quand il est justement interrogé sur l'adjonction des garçons, répond, sans équivoque, que le souci du gouvernement est de viser «les pédérastes $»^{41}$. Lors de la présentation du projet de loi devant les chambres,

\footnotetext{
${ }^{40}$ Commission extraparlementaire du Régime des mœurs, op.cit., Trentième séance, 13 avril 1906, p.762

${ }^{41}$ Commission extraparlementaire, op.cit. Séance du 30 novembre 1906, p.923. Louis Fiaux, op.cit., p.329-330. L'émissaire du gouvernement, Bulot évoque une documentation obligeant les pouvoirs publics à ne pas négliger la question. Pour parler de «cette vilaine matière», il cite les rapports d'inspecteurs sur les colonies pénitentiaires. Toujours pour éviter d'associer la prostitution à la masculinité, Louis Fiaux utilise des périphrases comme «l' autre prostitution » ou «l'autre sexe ».
} 
le représentant du gouvernement justifiera l'étendue du projet de loi par la nécessité d'établir une complète égalité de traitement pour tous les « déchus de l'espèce ${ }^{42}$.

Après des mois de travail de la commission, le texte de Le Poittevin est le seul à inspirer un projet de loi au gouvernement. La place occupée par les mineurs dans le débat est un nouvel indice de l'individualisation des mineurs dans le droit et les politiques à l'époque. Dans une société libérale, l'enfant, le jeune deviennent des objets autonomes des politiques publiques. Le projet devient loi. Le travail de la commission n'est donc pas entièrement caduque. A l'époque, le relatif consensus autour de la protection des mineurs est aussi un dérivatif pour taire l'échec d'une expertise plus globale sur le statut de la prostitution en France et satisfaire une partie de l'opinion.

\section{Le choix du politique. De la validation de l'expertise à son procès}

L'expertise de Le Poittevin et le texte de la sous commission vont servir d'appui à la politique du gouvernement. Alors que la commission tarde à produire un texte, le sénateur Bérenger, mis en minorité, a bien tenté de court-circuiter le travail des experts en déposant sa propre proposition au Sénat ${ }^{43}$. Pour parer le problème, en urgence, le gouvernement presse la commission de conclure. Son émissaire dépose un projet qui reprend, dans les grandes lignes, le texte voté par la sous-commission. La légitimité est double : celle du gouvernement et celle des experts dont l'étude a été requise comme solution possible à un problème ayant suscité une forte émotion dans la société civile. Porté par le gouvernement Clemenceau, le texte entend être l'expression d'une conscience républicaine et radicale sur des problèmes clairement identifiés comme sociaux. Dans le débat parlementaire, qui a été analysé par l'historien Jean Jacques Yvorel, les émissaires du gouvernement défendent un projet de loi « relative à la prostitution des mineurs ». L'argumentaire est précis : assurer la répression de la prostitution des mineurs de 18 ans et édicter des mesures de natures à obtenir le «redressement moral des mineurs ». Le projet est présenté aux représentants du peuple comme un texte de rupture avec «les bonnes mœurs ${ }^{44}$. L'année précédente, Clemenceau a reculé devant la proposition de loi de Paul Meunier portant sur l'abolition de la police des mœurs. Le texte était pourtant directement inspiré du travail de la Commission. Le projet sur les mineurs est là pour exprimer la position du gouvernement sur un terrain moins miné par

\footnotetext{
${ }^{42}$ cité par JJ Yvorel..., à partir du JO. Sénat, Documents parlementaires, 18 sept 1907, annexe n50, p.39.

${ }^{43}$ J-J Yvorel, op.cit. Voir également Anne Segretain, op.cit.

${ }^{44}$ A noter le recul de Clemenceau devant la proposition de loi de Paul Meunier sur l'abolition de la police des mœurs... en 1907. Texte pourtant directement inspiré du travail de la Commission.
} 
une contre-offensive des milieux néo-réglementaristes ${ }^{45}$. Le texte associe bien à l'article sur la prostitution, un second sur la débauche, une catégorie non définie sur un plan juridique, mais «catégorie du dialogue » pour une plus large captation des mineurs et un contrôle des sexualités déviantes.

Devant les parlementaires, les émissaires du gouvernement entendent distinguer la position du gouvernement radical des options défendues par un républicain conservateur comme René Bérenger. Face aux sénateurs, le 30 mars 1906, la parole est forte : « Si l'on n'envisageait que la prostitution sur la voie publique, l'oeuvre serait indigne d'une vraie démocratie ». Le projet de loi est une pierre supplémentaire dans l'élaboration d'une justice des mineurs qui, parce que le jeune est davantage perçu comme une victime, fait du tribunal civil, une instance de régulation des mesures qui, sous le couvert de protection, autorise le juge à le faire enfermer pour plusieurs années. Alors que différents scandales affectent l'image des établissements dits de préservation ou de réforme, que l'opinion a pu montrer son émotion devant le sort réservé à l'enfance populaire dans des établissements confessionnels visés par les campagnes des journaux républicains, les parlementaires interviennent pour plus de garanties afin que la liberté des sujets citoyens que sont les mineurs ou, plus encore, celle de leurs parents ne soient pas bafouées par un droit discrétionnaire ${ }^{46}$. Cette pression dit le souci des politiques de considérer les classes populaires, non plus comme des entités marginales, mais de penser, par les régulations juridiques, leur intégration dans la Nation. La mise à l'honneur du droit positif dans le champ de l'expertise et la législation signifient une inflexion, par la norme, dans le régime de suspicion à partir duquel, dans les années 1880 et 1890, la loi était fabriquée.

En 1908, la loi est votée. Dans les mois qui suivent, et l'analyse sera reprise dans les décennies suivantes, elle est présentée comme l'exemple même de la loi ratée parce que peu appliquée car inapplicable. Les débuts sont particulièrement laborieux. Comme les autorités publiques s'avèrent incapables de mettre en place les établissements spéciaux qui devaient prendre en charge une population que beaucoup jugent non réformable, le décret d'application est renvoyé en 1909, puis en 1910. Des études voient rapidement le jour pour proposer leur interprétation de «l'échec de la loi ». Bérenger est un des premiers à monter au créneau. A l'époque, il poursuit son rapprochement avec les idées défendues par Raymond Saleille, un des pères de la doctrine juridique de la défense sociale. L'analyse qu'il produit est directe : le

\footnotetext{
${ }^{45}$ En France, la cause des abolitionnistes doit attendre le second XX ${ }^{\circ}$ siècle pour être satisfaite : 1946 avec la suppression des maisons close. En 1960, la réglementation administrative est supprimée. Amélie Maugère, op.cit., p.11.

${ }^{46} \mathrm{~L}$ 'article 3 mentionne trois procès verbaux de constats de provocation à la débauche par le mineur.
} 
texte est inapplicable parce que la loi s'appuie sur la liberté individuelle du mineur. L'échec est le symbole des impasses de la doctrine du droit libéral classique dans la quête d'un ordre social protecteur ${ }^{47}$.

En 1914, le juriste Pierre de Casabianca est chargé de proposer une révision de la loi. Il estime la mission impossible. La loi serait trop viciée pour être aménagée. Son avis est tranché : l'échec actuel et futur d'une loi qu'il juge irréformable viendrait de sa dimension «trop politique $»^{48}$. Au regard de l'histoire sainte de la fabrique de la loi, le jugement aurait été une provocation en 1908. Il ne l'est plus dans le contexte de 1914. Le recul qu'autorise la pratique judiciaire et l'immersion dans un nouveau contexte politique la rendent pertinente. La fabrique de la loi s'est appuyée sur un monument d'expertise : le long et fastidieux travail de la commission extra-parlementaire. Le politique a pu donner l'impression de s'en remettre à la parole experte du juriste dans un contexte de forte émotion. Les savoirs et leurs normes sont des constructions qui n'échappent pas un état social et politique à partir duquel des collectifs ou des individus pensent le vrai, l'utile et le juste. Des hommes de la République radicale, parce qu'ils cherchaient une troisième voie, ont inspiré une philosophie de l'histoire, des idées sur le changement social qui ont défini un champ pour la réforme. La prostitution a été un territoire parmi d'autres. Dans ce cadre, l'extériorité des experts à l'égard des luttes partisanes, pourtant facteur de légitimité dans le savoir expert, a été toute relative.

A la veille de la guerre de 1914, alors que la France a traversé le «moment apache » de son histoire des jeunes, la production d'un discours sur l'échec de la loi dit les impasses de l'expertise dans la repensée d'un droit pour une protection de la jeunesse et la quête d'un modèle néophilanthropique pour remplacer les bonnes moeurs. Les difficultés ont été, dans les pratiques, de dépasser un modèle pénal de prise en charge des déviances et dans la norme, de faire du droit civil un outil opérationnel de changement dans la situation du mineur dit en danger. Les difficultés sont venues du refus du secteur privé ou des acteurs de l'assistance

\footnotetext{
${ }^{47}$ Pierre de Casabianca, Rapport sur les modifications à apporter à la loi du 11 avril 1910 pour en permettre l'application. Ministère de l'Intérieur. Commission chargée d'étudier les mesures destinées à assurer l'application de la loi du 11 avril 1908 sur les prostitués mineurs, Melun, impr. Administrative, 1919, 57p.

La loi, par sa simple existence et sa non application, est accusée de faciliter la prostitution des mineurs. L'auteur du rapport conclut que la loi «produit ce singulier résultat de faciliter la prostitution des mineurs au lieu de l'entraver ».La critique est reprise à la chambre. Voir, par exemple, les débats au Sénat, 2 février 1912 : la loi serait devenue «l'institution de la prostitution officielle de la jeunesse à Paris ».

Voir l'action de l'avocate Maria Vérone étudiée par Christine Machiels pour que les mineurs ne puissent être traduits en correctionnelle sous l'inculpation de vagabondage conformément à l'article $270 \mathrm{du}$ Code Pénal Christine Machiels, « «Protégeons la jeunesse ! » Maria Vérone, une avocate féministe face à la prostitution des mineur(e)s (1907-1938», RHEI,op.cit, p.119-137.

${ }^{48}$ De Casabianca est alors substitut du Procureur Général près de la Cour d'Appel de Paris. Entre 1908 et le 31 mai 1914, la loi a été appliquée à 156 mineurs dont 106 ont comparu devant le tribunal de la Seine. Pierre de Casabianca, op.cit. p.2.
} 
publique de s'impliquer dans une rééducation trop souvent pensée comme impossible de ceux qui, par le commerce du sexe, ne pouvaient plus être des enfants. Malgré l'expertise ou à cause de l'expertise, la représentation de l'échec informe sur le ressenti devant la difficulté à susciter une action publique en direction de mineurs qui, aux yeux de la majorité, demeurent marqués par la faute car ayant un comportement en marge des représentations de genre et d'âge associées à l'enfant victime ${ }^{49}$.

Les contemporains ont disserté sur l'échec de la réforme à court et moyen terme. Le recul autorise une lecture moins négative. La loi de 1908 a valeur d'étape dans un processus à plus longue échéance. Fruit d'un accommodement entre les intérêts experts et la volonté politique, la loi est un jalon dans la «moralisation» de la question prostitutionnelle ${ }^{50}$. La commission extraparlementaire a été une tribune pour un «discours juridique sur le sexe » et pour la promotion d'une « vision interventionniste, au nom de la protection des faibles et de leur dignité » que l'on retrouve dans les politiques ultérieures ${ }^{51}$. La loi de 1908 promeut et même banalise une régulation au civil des comportements juvéniles qui ne peuvent être atteints par le droit pénal. 1958 marquera le vrai changement avec l'adoption d'une ordonnance faisant de la protection judiciaire de l'enfance et de l'adolescence en danger, le pilier d'une justice des mineurs enfin individualisée.

\section{Pascale Quincy-Lefebvre (CERHIO UMR 6258-Université d'Angers)}

Agrégée et docteure en histoire, Pascale Quincy-Lefebvre est Maître de conférences en histoire contemporaine à l'université d'Angers (PRES UNAM). Elle est actuellement membre de Centre de Recherches Historiques de l'Ouest et fait partie du comité de rédaction de la Revue d'Histoire de l'Enfance "Irrégulière". Le Temps de l'histoire. Spécialiste d'histoire sociale, elle travaille actuellement sur " Normes, politiques et pratiques autour de l'enfance irrégulière" ainsi que sur la stratégie de "cause " dans l'histoire de la protection de l'enfance.

\footnotetext{
${ }^{49}$ Les impasses d'une action envers les « jeunes » au nom de la «protection de l'enfance » avec comme solution un enferment tout carcéral. Voir les recherches de Véronique Blanchard. L'auteure s'intéresse à la pratique des tribunaux mais également l'imaginaire « scientifico-juridique » les concernant. Elle estime qu'il « faut attendre le milieu des années 1970 pour que le thème de la prostitution soit abordé de manière différente par les acteurs sociaux. Le regard sur la place de la jeune fille évolue, les catégories se déplacent, les filles «perdues » perdent leur statut de perverses pour ne garder que celui de victimes», in «Les filles «perdues » sont-elles amendables ? Les mineures prostituées devant le tribunal pour enfants de la Seine dans les années cinquante », RHEI, op.cit.p.55.

${ }^{50}$ Sur la question plus générale des politiques sexuelles, Marcela Iacub, Le crime était presque sexuel et autres essais de casuistique juridique, Epel, Paris, 2002.

Sur le recul du standard des bonnes mœurs et son remplacement par la valeur de dignité humaine », Dominique Fenouillet, «Les bonnes mœurs sont mortes ! Vive l'ordre public philanthropique », Le droit privé français à la fin du XIXe siècle française à la fin du XXe siècle, Litec, Pais, p.487-528.

Voir également Bénédicte Lavaud-Legendre, Où sont passées les bonnes mours? Paris, PUF, 2005. p.69 La disparition de la notion des bonnes mœurs est présentée comme la conséquence de l'événement, en matière de mœurs, d'une protection juridique accrue des intérêts particuliers ...La notion des bonnes mœurs a été concurrencée par la vie privée.

${ }^{51}$ Daniel Borillo, Danièle Lochak, dir ., La liberté sexuelle, Paris, PUF, 2005.
} 
Mots clefs : Mineur[e]s, prostitution, déviance, régulation, expertise, juristes

\section{Résumé en français :}

Début XXe siècle, de nouveaux scandales mettent en cause l'administration policière de la prostitution en France. Une commission extraparlementaire est réunie pour réfléchir à un encadrement par la loi. Le système réglementaire n'est pas aboli mais une loi est votée en 1908 «sur les mineurs se livrant habituellement à la débauche ou à la prostitution ».

La commission (1903-1906) a fait une place importante à l'expertise des juristes sur des questions jusque là dominées par le savoir des médecins. Le changement tenait au contexte : la mobilisation républicaine autour des libertés après l'affaire Dreyfus. Le déplacement s'opéra également parce que la question des mineurs (filles et garçons) s'individualisait dans le droit. La « débauche » et la «prostitution » des mineurs sont alors posées comme des enjeux pour une politique judiciaire de «protection de l'enfance en danger». L'expertise justifia la nécessité de la loi. Les débats révélèrent également des divisions, entre juristes, sur les cadres de la loi, la nature du droit et le rôle des institutions judiciaires dans le contrôle de la sexualité des mineurs.

\section{Résumé en anglais :}

The early $\mathrm{XX}^{\text {th }}$ century is characterized by new scandals involving the Police administration in France. An extra-parliamentary commission is gathered to discuss the issue of establishing a legal framing. Although the statutory system is not abolished, a law is passed in 1908 about "the underage citizens who are used to indulge in debauchery or in prostituting themselves".

The commission (1903-1906) attached much importance to the jurists 'judgment about issues that were so far dealt with by doctors. This major change had to do with the historical context: the republican mobilization on the matter of individual liberties after the Dreyfus affair. It also occurred because the concern about underage citizens (boys and girls) "who [indulged] in debauchery or in prostituting themselves" was then becoming one of the most important issues in a judicial policy of "protecting endangered children". The jurists' judgment justified the necessity of legislating. The debates also emphasized the rifts between the jurists on the legal frames, on the choice between penal and civil right and on the role of the judicial institutions in controlling the minors' sexuality. 